\title{
Magnetite Particle Presence in the Human Brain: A Computational Dosimetric Study to Emphasize the Need of a Complete Assessment of the Electromagnetic Power Deposition at $3.5 \mathrm{GHz}$
}

\author{
David Vatamanu \\ Faculty of Engineering, Lucian Blaga University \\ and Nicolae Balcescu Land Forces Academy \\ Sibiu, Romania \\ davidvatamanu@yahoo.com
}

\author{
Simona Miclaus \\ Nicolae Balcescu Land Forces Academy \\ Sibiu, Romania \\ simo.miclaus@gmail.com
}

\begin{abstract}
The growing evidence of increased magnetite nanoparticles (both endo- and exogenic) in the human brain raises the importance of assessing the entire power deposition when electromagnetic waves at $\mathrm{GHz}$ frequencies propagate in such tissues. This frequency range corresponds to many popular portable communication devices that emit radiation close to a human's head. At these frequencies, the current dosimetric numerical codes can not accurately compute the magnetic losses part. This is due to the lack of an implemented computational algorithm based on solving the coupled Maxwell and LandauLifshitz-Gilbert equations, in the case of magneto-dielectrics, considering eddy currents losses and specific properties of magnetic sub-millimetric particles. This paper focuses on analyzing the limits and the inconsistencies when using commercial dosimetric numerical software to analyze the total absorbed power in brain models having ferrimagnetic content and being exposed to $3.5 \mathrm{GHz}$ electromagnetic waves. Magnetic losses computed using Polder's permeability tensor as constitutive relation lead to unreliable results. However, using such software can provide a preliminary view of the electromagnetic impact of ultra- and super-high frequencies on magnetic-dielectric tissues.
\end{abstract}

Keywords-magnetic brain; magnetite particles; magneticdielectric; microwaves dosimetry; power loss density

\section{INTRODUCTION}

Magnetic nanoparticles in human tissues, and more specifically in the brain, have been scarcely investigated for their electromagnetic wave absorption at Ultra-High Frequencies (UHF) $[1,2]$. The presence of biogenic magnetite $\left(\mathrm{Fe}_{3} \mathrm{O}_{4}\right)$ nanocrystals in the human brain was discovered in 1992 , while the concentration of $0.2-12 \mu \mathrm{g}$ magnetite in each $1 \mathrm{~g}$ of dry cerebral tissue was identified in 2009 [3]. Besides the biogenic magnetite particles, exogenic magnetite nanocrystals, arising from atmospheric pollution, were recently discovered to be deposited in some organs. These nanocrystals were first identified in 2016 in the human brain [4] and later in other organs too (heart, blood, liver, etc.) $[5,6]$. The exogenic magnetite nanoparticles present a different morphology from the endogenic [7]. Significant concerns were raised for increased health risks as these nanoparticles are regarded as highly toxic [8]. They can enter the blood flow and be transported to the brain. Moreover, they can catalyze the generation of reactive oxygen species in vivo and cause protein modification, lipid peroxidation, or DNA damage [8]. The increased magnetite content in the human brain raises another question about its contribution to the mechanism of thermal or non-thermal interactions with electromagnetic fields in high frequencies. In general, magnetite nanoparticles absorb very well electromagnetic waves at UHF frequencies [9-12]. Although their role in brain functioning is still unknown, memory mechanisms' [13] or crossing the blood-brain barrier [14-15] involvements cannot be neglected. A map of locations and mass information about magnetite nanoparticles present in the human brain was published in 2018 [16], while the concentration differences in the brains of people belonging to different geographical regions were highlighted in 2021 [17]. To answer this question and address the problem of quantification of electromagnetic power deposition in tissues having ferro- or ferrimagnetic content, a simulation was conducted on wave propagation in human brain models using commercial software. Such software suites provide radiofrequency/microwave dosimetry solutions at high frequencies but have a serious limitation regarding their capability to compute correctly the magnetic losses.

The electromagnetic waves emitted by various communication devices using UHF or Super-High Frequency (SHF) bands affect the human head in a very significant manner. Due to the eyes, ears, and mouth positions, the brain is practically always near the wave sources during all kinds of wireless communication devices usage. The brain is a target of electromagnetic radiation and the dose rate it receives has various thermal and non-thermal biological effects. The thermal effects represent the basis of the human protection standards metrics and safety limits [18, 19], while the nonthermal are still under debate and study [20-22]. On the other 
hand, radiofrequency and microwave numerical dosimetry is continuously following the development and the popularity of emitting technologies [23-30]. Computational dosimetry using pure dielectric head models started more than 20 years ago and developed progressively using more and more accurate head models [31-40]. However, none dosimetric research provided a comparative quantification of the dissipated power when a brain contains magnetite or not. In this respect, this work underlines the limits and gaps in conducting a realistic quantification of the entire power loss in a "magnetic brain" by using available software, emphasizing its possible role to address the "magnetic brain" dosimetric problem in the future.

Micromagnetic software suites are widely used in the electronic industry, but they can't be utilized in bioelectromagnetics, as they generally consider tissues as pure dielectrics. These software suites have not implemented the proper coupled Maxwell and Landau-Lifshitz-Gilbert (LLG) equations to provide reliable results concerning the magnetic power losses when very high-frequency waves pass a tissue containing magnetic particles. Neither the eddy currents nor the specific properties (geometric and magnetic anisotropies, etc.) of the magnetic nanoparticles are considered in such approaches. This analysis introduces the subject of reconsidering the software to use in order to assess the real impact of the magnetic nanoparticles' presence in tissues.

\section{LIMITATIONS OF PRESENT RADIOFREQUENCY SOFTWARE}

The CST Studio Suite [41] is used to simulate and analyze antenna parameters [42-44]. CST and all similar commercial software provide also a monitor function for computing the Specific Absorption Rate (SAR) of energy deposition in the tissues. SAR is a reference metric in all standards and is defined by taking into account only the dielectric losses in the tissues. SAR is related to temperature increase $(\Delta T)$ by:

$$
\mathrm{SAR}=\frac{\sigma E_{r m s}^{2}}{\rho}=c \frac{\Delta T}{\Delta t}
$$

where $\sigma$ is the electric conductivity of the tissue, $E_{r m s}$ is the root-mean-square value of the internal/local electric (E)-field strength, $\rho$ is the mass density, $c$ is the specific heat of the tissue, $\Delta T$ is the temperature variation, and $\Delta t$ is the time duration of the wave propagation that dissipated the heat. As SAR is expressed in $\mathrm{W} / \mathrm{kg}$ and power loss density is expressed in $\mathrm{W} / \mathrm{m}^{3}$, they can be directly connected to the mass density.

Magnetic losses are not included properly in SAR calculations of current dosimetric software packages. Magnetic losses may have 4 possible origins: a) relaxation due to the rotation of the magnetic moments (without rotation of the whole particle) - Neel relaxation, b) relaxation due to the rotation of the particle in the alternating field - Brownian relaxation, c) shifting of magnetic domain walls in multidomain materials - hysteresis loss, and d) generation of Foucault (eddy) currents in bulk materials or at centimetric scale (resistive heating when the magnetic flux is rapidly varying) [45-48]. The first 3 mechanisms of heat dissipation are detected mainly in nanometric dimensions and depend on size, shape, crystalline anisotropy, morphology, and degree of aggregation/agglomeration of the crystals. If ferro- or ferrimagnetic (like magnetite) material is present in a dielectric tissue, the whole organ should be treated as a magnetodielectric. Sub-millimetric magnetic particles are called micromagnetics. "Magnetic brain" falls in the magnetodielectrics and should be treated by micromagnetics, since the dimensions of the magnetite crystals are less than $200 \mathrm{~nm}$ if exogenic and less than $70 \mathrm{~nm}$ if endogenic. The dynamics of the magnetization vector $M$ in a material exposed to an alternating magnetic field $H$ is governed by the LLG equation [49]:

$$
\frac{\partial M}{\partial t}=-\frac{\gamma}{1+\alpha^{2}} M \times H_{e f f}-\frac{\gamma \alpha}{\left(1+\alpha^{2}\right) M_{s}} M \times M \times H_{e f f}
$$

where $M_{s}$ is the saturation magnetization, $\alpha$ is the damping constant, $\gamma$ is the gyromagnetic ratio of the electron, and $H_{e f f}$ is the effective magnetic field. $H_{\text {eff }}$ includes the actual magnetic field $H$ obtained as a solution of the Maxwell equations and other terms which take into account the crystalline anisotropy, the Heisenberg exchange interaction (quantum mechanical effect), etc. The LLG equation states that the change rate of the magnetic moment (magnetization $M$ ) is proportional to the torque due to different interactions. The magnetic moment itself is due to the orbital rotation and spin of electrons. The Larmor precession of the spin creates a resonant frequency in the magnetic material property, defined by ferromagnetic resonance. Equation (2) shows that torques are due to the magnetic field acting on magnetic moments. So the LLG equation requires knowledge of the magnetic field, which can be obtained by solving the Maxwell equations.

The vast majority of micromagnetic solvers use the magnetostatic approximation to the Maxwell equations [41]. In that case, E- and H-fields are completely decoupled. Many micromagnetic software suites solve the LLG equation dynamically with coupling to the magnetostatic solution [49, 50]. These tools do not predict the interaction between the magnetization $M$ and electromagnetic waves. In that case, the Maxwell equations do not include any dynamic effects, and the fields propagate through the system instantaneously. The static approximation is valid when $\tau_{m} / \tau \ll 1$, where $\tau$ is the characteristic time of the system dynamics, and $\tau_{m}$ is the magnetic diffusion time constant given by $\tau_{m}=\sigma \mu L^{2}$, where $\mu$ is the magnetic permeability and $L$ is the system size [51].

When frequency increases, the effects of induced eddy currents and dynamic magnetic fields become significant and cannot be neglected. In such cases, it becomes necessary to renounce the static Maxwell equations [50-55]. The magnetoquasistatic approximation (eddy current approximation) of the Maxwell equations is based on neglecting the displacement current term $\partial \mathrm{D} / \partial t$. Eddy current effects are negligible only when time variations of $H$ and $M$ are slow enough and the system size is smaller than the skin depth. A magnetoquasistatic approximation is therefore valid whenever the system size is much smaller than the wavelength, and when the induced E-fields (due to time-varying $\mathrm{H}$-fields and $M$ ) are the dominant effect over capacitive effects. This approximation neglects wave propagation effects and raises the diffusion of both $\mathrm{H}$-field and induced eddy currents. Indeed, under the magneto-quasistatic approximation, $\mathrm{H}$-field satisfies the following diffusion equation [49]:

$$
\frac{\partial H}{\partial t}=\frac{1}{\sigma \mu_{0}} \nabla^{2} H-\frac{\partial M}{\partial t}+\frac{1}{\sigma \mu_{0}} \nabla \nabla \cdot M
$$


A similar equation is valid for the induced eddy currents, noted by $J$.

Full-wave electromagnetic simulators, such as CST Studio Suite and Ansys HFSS 3D High-Frequency Simulation Software [56], solve Maxwell's equations by defining material dispersion and anisotropy through the application of Polder's permeability tensor as the constitutive relation. This tensor is used because ferrimagnetic materials become anisotropic in the presence of a magnetizing field. From the perspective of solving the Maxwell equations properly, the LLG acts as the constitutive relation between the magnetic field $H$ and the magnetic flux density $B$ through $B=\mu_{0}(H+M)$. Given $H$, the magnetization $M$ is obtained by solving the LLG equation. The LLG and Maxwell equations must be solved jointly as a coupled system of differential equations, for realistic and complete computation results. Different strategies can be adopted to solve the coupled Maxwell-LLG equations. A coupled micromagnetic-Maxwell equations solver was developed only recently, taking into account the effect of the eddy currents $[49,51]$. Neglecting the effects of eddy currents in the $\mathrm{GHz}$ spectrum is completely inappropriate. An efficient and accurate modeling tool for multiphysics problems that encompasses electrodynamics and micromagnetics was proposed in [48]. Three major problems in modeling interactive micromagnetics and electromagnetic waves were solved using [48]: 1) coupling between Maxwell's equations and the LLG equation in every time step, 2) scale disparity of the multiphysics, 3) field discontinuity at material interfaces. However, such advanced software packages [52, 55] have not yet been implemented to treat bioelectromagnetic dosimetry issues. In the multiphysics problem, involved in ferro- and ferrimagnetic materials exposed to radiofrequency/microwaves, the dynamic Maxwell's (4) and LLG (5) equations must be considered as:

$$
\begin{gathered}
\nabla \times H=\varepsilon \frac{\partial E}{\partial t}+J+\sigma E \\
\nabla \times E=-\frac{\partial B}{\partial t} \\
\frac{\partial M}{\partial t}=\mu_{0} \gamma(M \times H)-\frac{\alpha}{|M|} M \times \frac{\partial M}{\partial t}
\end{gathered}
$$

Consequently, using CST Studio for dielectric versus magnetic brain simulations provides an incomplete/partial solution to the role that magnetite crystals have in absorbing electromagnetic waves in the UHF and SHF spectra since it doesn't implement the coupled Maxwell-LLG equations. Further on, CST Studio was used with its underlined limitations to quantify power loss, stored electric energy, and magnetic energy densities in pure dielectric versus magnetodielectric brain models. The absorbed power (equal to power loss $P_{\text {loss }}$ ) was calculated in each model by using the mean power loss density values and the corresponding volumes of the materials. The energy density formula is obtained as a byproduct of the energy conservation law (Poynting theorem), which is derived using Maxwell's equations with the aid of the equations of motions of the polarization $P$ (in dielectric) and magnetization $M$ (in magnetic) mediums:

$$
-\nabla \cdot \mathrm{S}=\frac{\partial W}{\partial t}+P_{\text {loss }}
$$

where $S, W$, and $P_{\text {loss }}$ stand for the Poynting vector (power density in $\mathrm{W} / \mathrm{m}^{2}$ ), energy density (in $\mathrm{J} / \mathrm{m}^{3}$ ), and power loss (W), respectively. Equation (6) can be re-written as:

$$
\begin{gathered}
-\nabla \cdot(\mathrm{E} \times \mathrm{H})=\frac{\partial w_{e}}{\partial t}+\frac{\partial w_{b}}{\partial t}+P_{\text {loss }} \\
P_{\text {loss }}=\text { E. J }
\end{gathered}
$$

where $W_{e}$ and $W_{b}$ are the electric and magnetic energy densities, and $J$ is the current density. Equation (7) can be rewritten as:

$$
\begin{aligned}
-\nabla \cdot(\mathrm{E} \times \mathrm{H})=\mathrm{E} \cdot \frac{\partial \mathrm{D}}{\partial t}+\mathrm{H} \cdot \frac{\partial \mathrm{B}}{\partial t} \\
=\frac{\partial}{\partial t}\left(\frac{\epsilon_{0} \mathrm{E}^{2}}{2}\right)+\mathrm{E} \cdot \frac{\partial \mathrm{P}}{\partial t}+\frac{\partial}{\partial t}\left(\frac{\mu_{0} \mathrm{H}^{2}}{2}\right) \\
+\mu_{0} \mathrm{H} \cdot \frac{\partial \mathrm{M}}{\partial t}
\end{aligned}
$$

The time-averaged energy density of a harmonic electromagnetic wave, $\langle W\rangle$, can be calculated as:

$$
\langle W\rangle=\frac{\epsilon_{0}}{4} \frac{\partial(\omega \epsilon(\omega))}{\partial \omega}|\mathrm{E}|^{2}+\frac{\mu_{0}}{4} \frac{\partial(\omega \mu(\omega))}{\partial \omega}|\mathrm{H}|^{2}
$$

III. COMPUTING THE ELECTROMAGNETIC LOSSES BASED ON POLDER'S PERMEABILITY TENSOR AS CONSTITUTIVE RELATION

\section{A. Computational Models: Geometries, Electric and Magnetic Properties}

Since no micromagnetic solver has yet the capacity to include eddy currents' effects, CST Studio was used for some magneto-dielectric material computations. A computational approach was applied, using magnetite particles having a radius of $1 \mathrm{~mm}$, to distinguish the contribution of these particles to the total absorbed power in the brain and separate the electric and magnetic energy densities. The computations were conducted in the frequency domain and used almost the same mesh dimensions in all cases (mesh properties: hexahedral, cells/wavelength $=15$, spherical brain model: smallest cell $=$ 0.2955 , largest cell $=5.6774$, realistic brain model: smallest cell $=0.3217$, largest cell $=5.6832$ ). A series of electromagnetic exposures were designed, computed, and analyzed to observe the contribution of the magnetite particles to the total absorbed power in the following brain models:

- S1: a simple spherical brain model (homogeneous), having the dimensions and material properties given in Table I. This model was purely dielectric.

- S2: a simple dielectric brain sphere, having a single particle of magnetite located in its center; magnetite volume was $\mathrm{V} 1=682.67 \mathrm{~mm}^{3}$;

- S3: a simple dielectric brain sphere, containing N1=163 uniformly spread identical smaller particles of magnetite. Their total volume was equal to V1.

- S4: a simple dielectric brain sphere, having N2=129 uniformly spread smaller particles of magnetite (identical). Their total volume was equal to $\mathrm{V} 2=540.27 \mathrm{~mm}^{3}$. 
- S5: an MRI-based anatomical human head model, with non-magnetic brain tissues inserted (head of Gustav, belonging to the Bio-Models library, $2.08 \times 2.08 \times 2 \mathrm{~mm}$ spatial resolution, containing 11 tissue types, 134028 voxels were used for the brain model while its mass was $1211.905 \mathrm{~g}$ ). The brain had the same volume as the simple spherical brain model (homogeneous).

- S6: an MRI-based anatomical human head model with magnetite inserted in the brain tissue (Gustav model with magnetite). A number $\mathrm{N} 2=129$ magnetite particles were uniformly spread in this model, with their total volume being equal to $\mathrm{V} 2$.

The mass concentration of magnetite in the brain was $\mathrm{C} 1=1.6 \mathrm{mg} / \mathrm{g}$ for $\mathrm{S} 2$ and $\mathrm{S} 3$, and $\mathrm{C} 2=2.34 \mathrm{mg} / \mathrm{g}$ for $\mathrm{S} 4$ and $\mathrm{S} 6$. Table I shows the dimensions of the materials used in the models. Table II presents the dielectric, magnetic, and other relevant properties of the materials at $3.5 \mathrm{GHz}$. Magnetite dielectric and magnetic parameters at $3.5 \mathrm{GHz}$ were extracted from [49], while the brain and the rest tissues of the head were modeled based on dielectric data presented in [50].

TABLE I. BRAIN TISSUE AND MAGNETITE PARTICLES DIMENSIONS

\begin{tabular}{|c|c|c|c|c|c|c|}
\hline $\begin{array}{l}\text { Exposure } \\
\text { situation }\end{array}$ & $\begin{array}{c}\text { Magnetite } \\
\text { Volume } \\
\left(\mathrm{mm}^{3}\right)\end{array}$ & $\begin{array}{c}\text { Brain } \\
\text { Volume } \\
\left(\mathrm{mm}^{3}\right)\end{array}$ & $\begin{array}{c}\text { Magnetite } \\
\text { Mass (g) }\end{array}$ & $\begin{array}{c}\text { Brain } \\
\text { Mass } \\
(\mathrm{kg})\end{array}$ & \begin{tabular}{|c} 
Brain \\
sphere \\
diameter \\
$(\mathrm{mm})$
\end{tabular} & $\begin{array}{c}\text { Magnetite } \\
\text { sphere } \\
\text { diameter } \\
(\mathrm{mm}) \\
\end{array}$ \\
\hline S1 & - & 2144661.00 & - & 2.24 & 160 & - \\
\hline S2 & 682.67 & 2143978.00 & 3.58 & 2.24 & 160 & 10.92 \\
\hline S3 & 682.67 & 2143978.00 & 3.58 & 2.24 & 160 & 2 \\
\hline S4 & 540.28 & 1159170.72 & 2.83 & 1.21 & 130.35 & 2 \\
\hline S5 & - & 1159717.70 & - & 1.21 & N/A & - \\
\hline S6 & 540.275091 & 1159177.43 & 2.83 & \begin{tabular}{l|}
1.21 \\
\end{tabular} & N/A & 2 \\
\hline
\end{tabular}

TABLE II. SIMULATION MATERIALS' PROPERTIES

\begin{tabular}{|l|c|c|c|c|}
\hline & Brain & Magnetite & Copper & FR4 \\
\hline Material type & Normal & Normal & $\begin{array}{c}\text { Lossy } \\
\text { material }\end{array}$ & Normal \\
\hline $\begin{array}{l}\text { Real part - relative } \\
\text { electric permittivity }\end{array}$ & 45.81 & 4.8 & N/A & 4.3 \\
\hline $\begin{array}{l}\text { Real part - relative } \\
\text { magnetic permeability }\end{array}$ & 1 & 0.84 & 1 & 1 \\
\hline $\begin{array}{l}\text { Electric Conductivity } \\
(\mathrm{S} / \mathrm{m})\end{array}$ & 0.767 & 0.233657 & $5.8 \mathrm{E}+07$ & 0.00861191 \\
\hline $\begin{array}{l}\text { Magnetic Conductivity } \\
(1 / \text { Sm) }\end{array}$ & 0 & 7428.26 & N/A & 0 \\
\hline $\begin{array}{l}\text { Thermal Conductivity } \\
(\mathrm{W} / \mathrm{K} / \mathrm{m})\end{array}$ & 0.5 & 5.5 & 401.0 & 0.3 \\
\hline Heat capacity $(\mathrm{kJ} / \mathrm{K} / \mathrm{kg})$ & 3.7 & 0.6 & 0.39 & 0 \\
\hline $\begin{array}{l}\text { Bloodflow coefficient } \\
\left(\mathrm{W} / \mathrm{K} / \mathrm{m}^{3}\right)\end{array}$ & 40000 & 0 & 0 & 0 \\
\hline $\begin{array}{l}\text { Basal metabolic rate } \\
\left(\mathrm{W} / \mathrm{m}^{3}\right)\end{array}$ & 7000 & 0 & 0 & 0 \\
\hline Mass density $\left(\mathrm{kg} / \mathrm{m}^{3}\right)$ & 1045 & 5240 & 8930 & N/A \\
\hline
\end{tabular}

\section{B. Characterization of Emitting Antenna and Field}

Electromagnetic waves were generated by a patch antenna (made of copper and FR4 dielectric). Its geometry and dimensions are presented in Figure 1(a), and it emitted a continuous wave on $3.5 \mathrm{GHz}$. This frequency is used in WiMAX communications, based on the IEEE 802.16, and in the first implementations of the $5^{\text {th }}$ generation $(5 \mathrm{G})$ mobile broadband communications worldwide. The simulated power of the patch antenna was $0.5 \mathrm{~W}$, but simulations in CST showed a Voltage Standing Wave Ratio (VSWR) of 1.27 due to its mismatch loss, and the radiated power from the antenna was $0.138 \mathrm{~W}$ due to its total efficiency of $28 \%$ (Figure $1(\mathrm{~b})$ ). The far-field radiation pattern of this antenna at $3.5 \mathrm{GHz}$ is presented in Figure 1(c). As it can be observed, the gain is low on the $\mathrm{O}-\mathrm{z}$ direction $(-7.8 \mathrm{dBi})$, therefore the exposure of the brain model is very low.

(a)

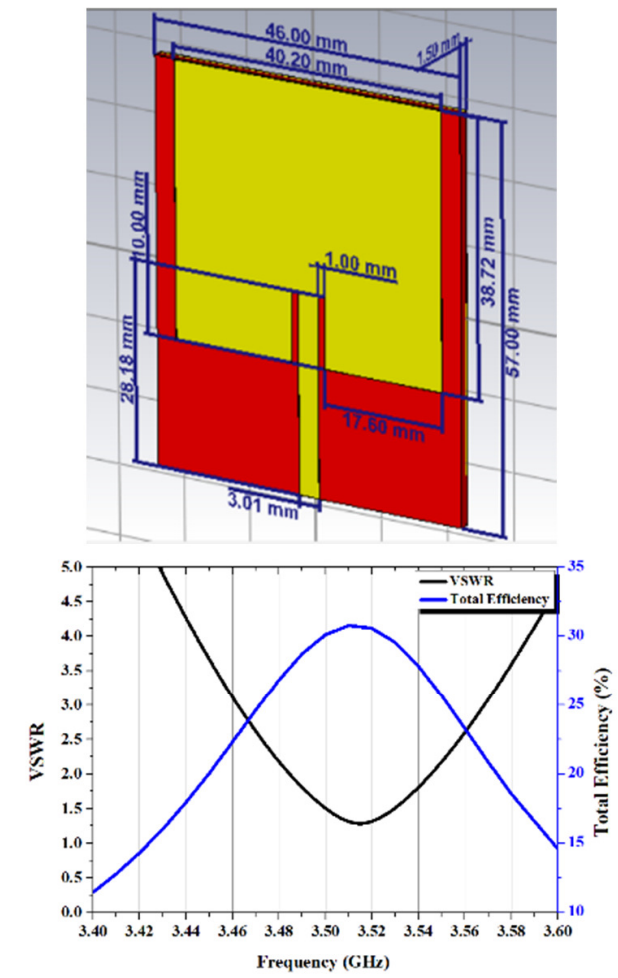

(b)

(c)

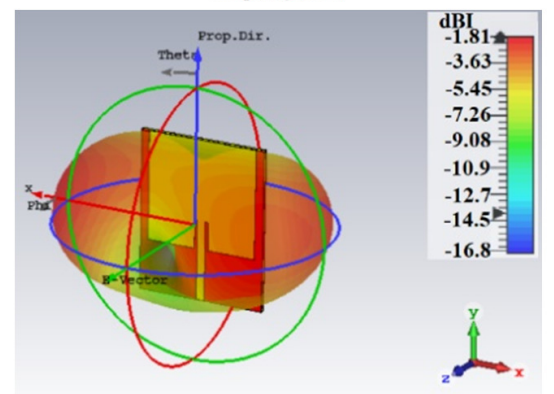

Fig. 1. The emitting patch antenna model: (a) geometric parameters, (b) stationary wave ratio and total efficiency at $3.5 \mathrm{GHz}$, c) $3 \mathrm{D}$ radiation pattern at $3.5 \mathrm{GHz}$.

\section{Exposure of Non-Magnetic and Magnetic Brain Models}

The geometries of the exposure models are shown in Figures 2 and 3. The distance between the antenna surface and the incidence point of the model (brain, in the case of a full head model) was always $45 \mathrm{~cm}$, therefore the far-field conditions of exposure were fulfilled in all cases. Figures 2(b)(c) show the positions of the magnetite particles in the simple 
homogeneous spherical model of the brain (S1, S2, S3, S4). Figures 3(b) and 4 show the magnetite particles distribution in the realistic brain model $(\mathrm{S} 5, \mathrm{~S} 6)$. The results of S1-S3 cases can be compared because the mass concentration of magnetite is the same $(\mathrm{C} 1)$. Similarly, the results of the S4-S6 cases can be compared due to the same concentration of magnetite (C2).

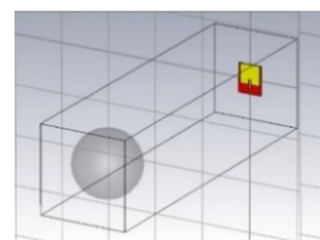

(a)

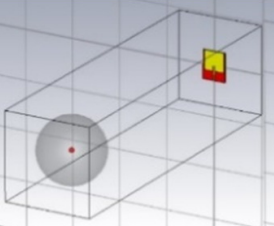

(b)

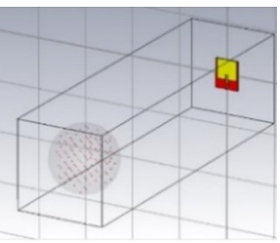

(c)

Fig. 2. The computational space figuring the positions of the antenna, simplified brain model (sphere) and the distribution of the magnetite particles: (a) non-magnetic brain, (b) brain containing a single magnetite particle, (c) brain containing spread magnetite particles with a total volume equal to the single-particle from situation (b).

(a)

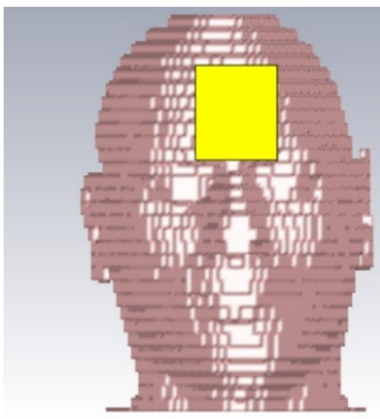

(b)

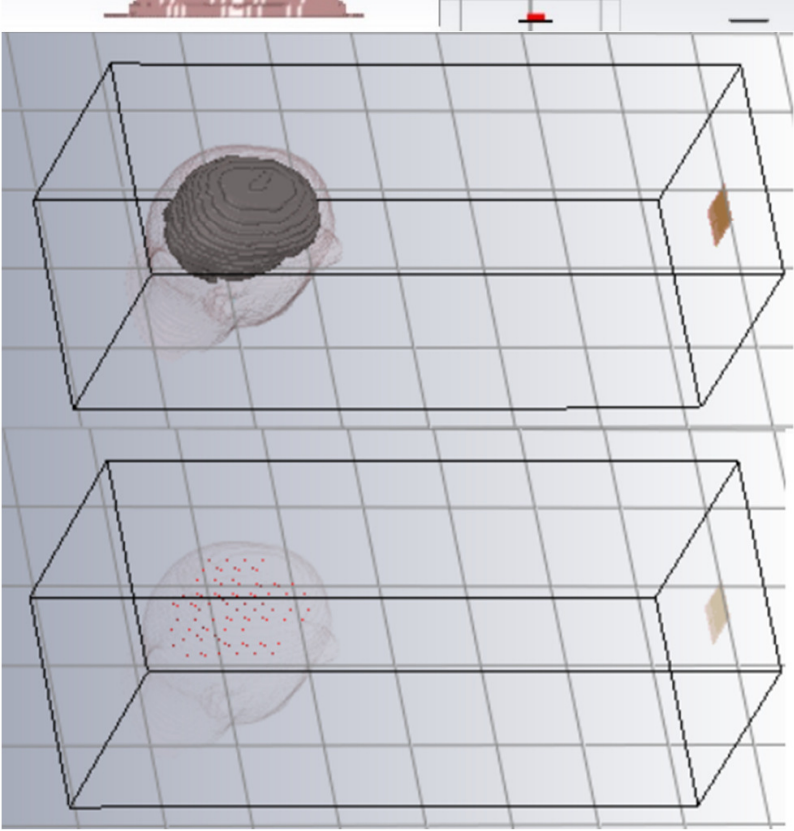

Fig. 3. (a) The human anthropomorphic head model (Gustav) and the positioning of the patch antenna, (b) figuring the non-magnetic brain in the head (up) and the distribution of the magnetic particles in the magnetic brain case.

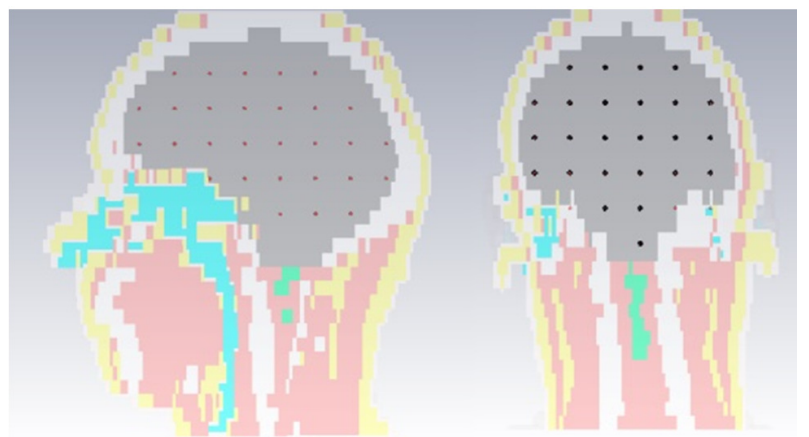

Fig. 4. Details of the distribution (homogeneous) of the magnetite particles in the realistic magnetic brain model.

\section{RESUlts}

Figure 5 shows the distribution of the E-field levels in air tangential to the head model. In all S1-S6 cases, the distribution and the absolute values of the E-field strengths in the coronal plane are very similar to Figure 5 . It can be observed that the incident field strengths are not exceeding $4.5 \mathrm{~V} / \mathrm{m}$ and they are mostly concentrated in the range between $1-3 \mathrm{~V} / \mathrm{m}$ in the coronal plane.

(a)
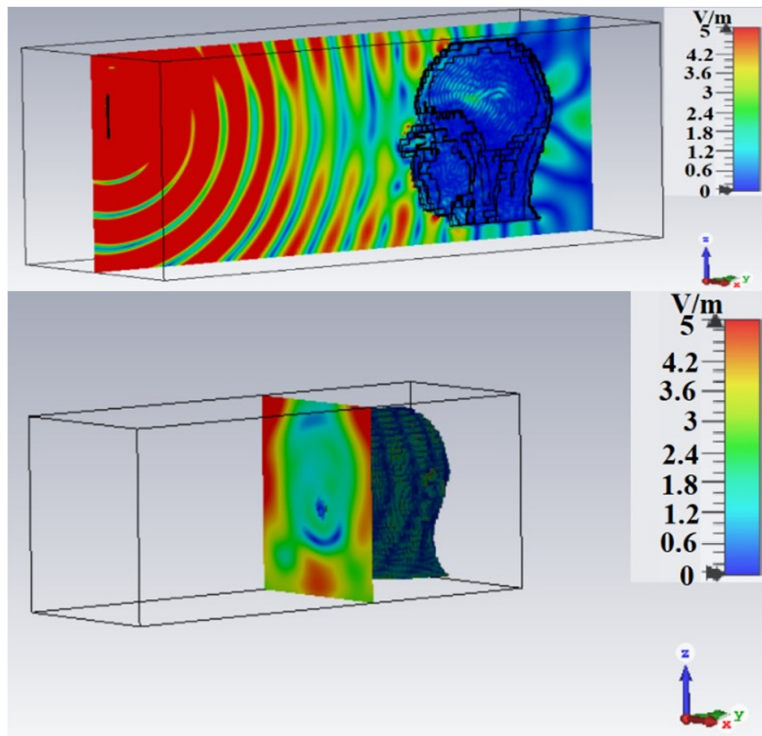

Fig. 5. Incident E-field strength in the air: (a) sagittal plane, (b) coronal plane tangential to the nose of the model.

Table III shows the following post-processed values: a) the mean and the maximum power densities in each model, b) the mean values of the electric and of the magnetic energy densities, c) the mean power loss in each model, d) the normalized power loss (the ratio between the mean power loss in the model and the mean E-field strength in the air, in the coronal plane tangent to the model surface), e) the normalized total SAR (the ratio between the total mean power loss per mass unit of the model and the mean E-field strength in the air, in the coronal plane tangent to the model surface). For example, comparing S3 with S1 suggests that power loss, its normalized value, and normalized SAR are lower when the 
magnetite is spread in the brain than when not present at all, which is an intriguing result. On the other hand, comparing S6 with S5, when the magnetite concentration is higher, power loss, its normalized value, and normalized SAR are higher when the magnetite is spread in the brain than when not present at all. Therefore, the presence of magnetic material seems not to enlarge the losses consistently.

TABLE III. EFFECTS OF MAGNETITE PRESENCE IN BRAIN EXPRESSED BY STORED ENERGY DENSITY AND LOSSES (INCLUDING NORMALIZED ONES)

\begin{tabular}{|c|c|c|c|c|c|c|c|c|c|}
\hline \multirow{2}{*}{ Case } & \multirow{2}{*}{$\begin{array}{l}\mathbf{E}_{\text {incident }} \\
(\mathbf{V} / \mathbf{m})\end{array}$} & \multirow{2}{*}{ Material type } & \multicolumn{2}{|c|}{ Power loss density $\left(\mathrm{W} / \mathrm{m}^{3}\right)$} & \multicolumn{2}{|c|}{ Mean energy density $\left(\mathrm{J} / \mathrm{m}^{3}\right)$} & \multirow{2}{*}{$\mathbf{P}_{\text {loss }}(\mu \mathrm{W})$} & \multirow{2}{*}{$\begin{array}{l}\text { Normalized power } \\
\operatorname{loss}(\mu \mathrm{W} /(\mathrm{V} / \mathrm{m}))\end{array}$} & \multirow{2}{*}{$\begin{array}{l}\text { Average normalized total } \\
\operatorname{SAR}((\mu \mathrm{W} / \mathrm{kg}) /(\mathrm{V} / \mathrm{m}))\end{array}$} \\
\hline & & & Max & Mean & Electric & Magnetic & & & \\
\hline S1 & 4.11 & Brain & 7.37 & 0.11 & $5.61 \mathrm{E}-11$ & $5.68 \mathrm{E}-11$ & 227.33 & 55.27 & 24.66 \\
\hline S2 & 4.12 & Brain & 6.77 & 0.11 & $5.61 \mathrm{E}-11$ & $5.67 \mathrm{E}-11$ & 227.49 & 55.27 & 24.63 \\
\hline \multirow{2}{*}{ S3 } & \multirow{2}{*}{4.01} & Brain & 7.30 & 0.10 & $5.35 \mathrm{E}-11$ & $5.40 \mathrm{E}-11$ & \multirow{2}{*}{216.70} & \multirow{2}{*}{54.03} & \multirow{2}{*}{24.08} \\
\hline & & Magnetite & 2.18 & 0.23 & $2.92 \mathrm{E}-11$ & $4.12 \mathrm{E}-11$ & & & \\
\hline S4 & 3.94 & Brain & 8.19 & 0.11 & $5.98 \mathrm{E}-11$ & $6.04 \mathrm{E}-11$ & 131.17 & 33.27 & 27.41 \\
\hline S5 & 3.97 & Brain & 2.25 & 0.06 & $8.47 \mathrm{E}-11$ & $8.47 \mathrm{E}-11$ & 70.74 & 17.82 & 14.70 \\
\hline \multirow{2}{*}{ S6 } & \multirow{2}{*}{3.96} & Brain & 2.23 & 0.06 & $8.40 \mathrm{E}-11$ & $8.40 \mathrm{E}-11$ & \multirow{2}{*}{70.89} & \multirow{2}{*}{17.89} & \multirow{2}{*}{14.76} \\
\hline & & Magnetite & 3.53 & 0.33 & $4.39 \mathrm{E}-11$ & $7.05 \mathrm{E}-11$ & & & \\
\hline
\end{tabular}

Figure 6 shows the sectioning plane orientation in the models that will be used for presenting the distributions in Figures 7-9. Figure 7 shows the distribution of the electric energy density stored in the models, in the chosen section plane, for all 6 cases. In S3 and S4 images, the magnetite particles are emphasized to observe the lower levels of energy density at their positions. S5 is the dielectric brain in the head model, while S6 is the magnetic brain in the head model. The position of the magnetite particles is still observable, as the energy density is lower in their positions than in their vicinity.

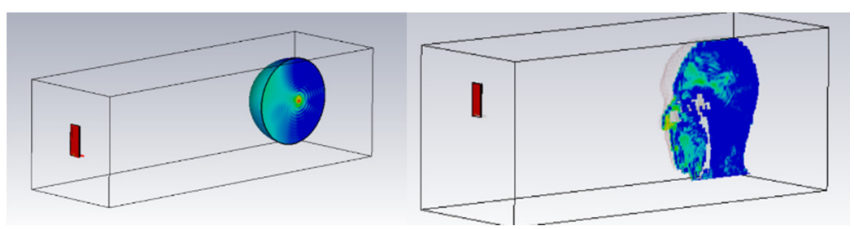

Fig. 6. Figuring the section plane applied to the models to show the spatial distributions of energy density and power loss density.
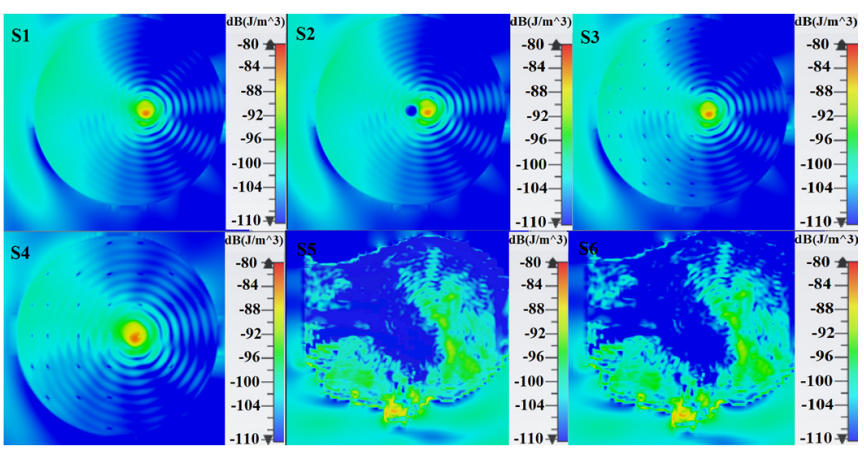

Fig. 7. Electric energy density distribution in the chosen sagittal section plane in the 6 cases.

Figure 8 shows the distribution of the magnetic energy density for all 6 cases. The position of the magnetite particles is still observable in S3 and S4, again, as a bit smaller values than in the neighboring material. No differences appear between S5 and S6 regarding the magnetic energy density accumulation in the dielectric versus the magnetic brain.
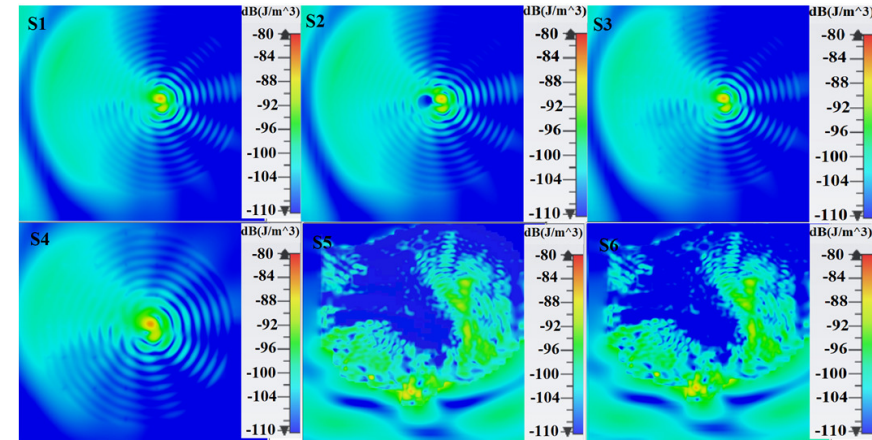

Fig. 8. Magnetic energy density distribution in the chosen sagittal section plane in the 6 situations.

Figure 9 shows the power loss density distribution map in the chosen section plane. The positions of the magnetic particles are delimited visually in all cases. Figure 10 shows an enlarged image for the Gustav head model of case S6. Even with the micromagnetic limits of CST, which is using Polder's permeability tensor to describe the magnetic properties at a larger scale and to compute the losses, obvious excess power deposition is due to the presence of magnetite when the $3.5 \mathrm{GHz}$ wave is propagating in the 11-tissue MRI human head model having magnetite particles.

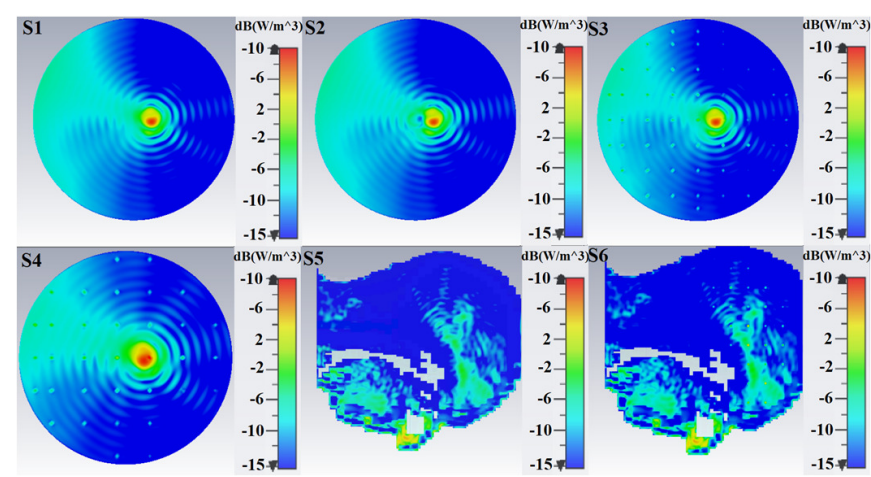

Fig. 9. Power loss density distribution in the chosen sagittal section plane in the 6 cases. 


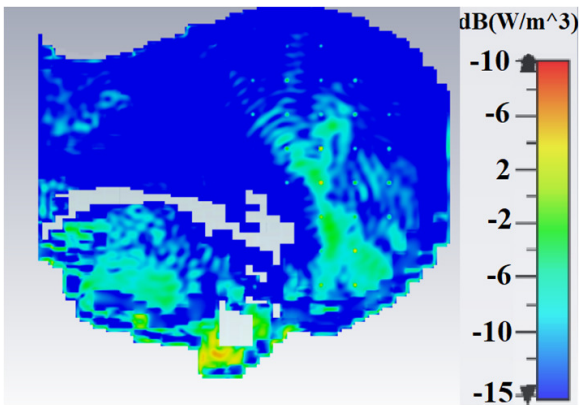

Fig. 10. Magnification of the S6 case to observe the losses due to magnetite particles present in the brain by using the computations based on Polder's permeability tensor as a constitutive relation.

Based on the results tabulated in Table III, emphasis was given to the overall differences between the models, regarding: a) the ratio between the magnetic and electric energy densities accumulated in each model, in tandem with its volume (Figure $11), \mathrm{b})$ the percentage the magnetic brain is lossier than the pure dielectric (Figure 12), and c) the power loss densities (max and average) in the magnetic and dielectric models (Figure 13). The results were grouped in two sections in each figure, corresponding to the two difference concentrations of magnetite $(\mathrm{C} 1, \mathrm{C} 2)$. Figure 11 shows that magnetite concentration is not determining the energy density ratio between the magnetic and electric energies, as possibly higher magnetite content results in lower stored magnetic energy density. Moreover, this ratio is larger in all magnetic brain models compared to purely dielectric. The overall concentration, distribution, and dimensions of magnetite particles matter, while the problem becomes more complex when examining geometric and magnetic anisotropies, particle geometry, local computation of $H_{\text {eff, }}$ etc.

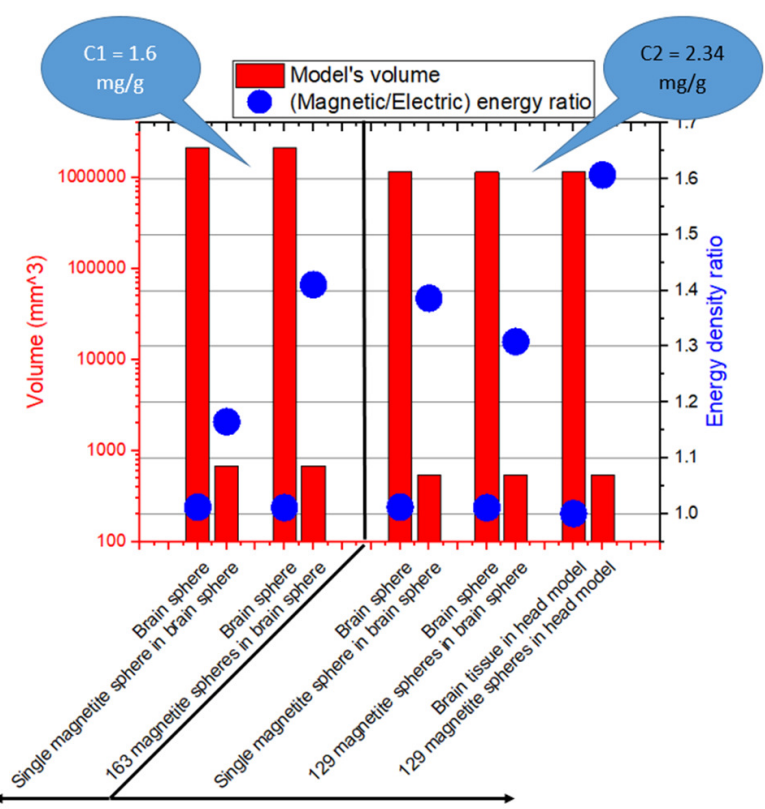

Fig. 11. The ratio of magnetic to electric energy densities stored in pure dielectric versus magnetic brain models (blue balls) in tandem with the volume of the models (red bars). Two categories are delimited due to two different concentrations of magnetite $(\mathrm{C} 1, \mathrm{C} 2)$.
Figure 12 shows that for the same concentration of magnetite $\mathrm{C} 1$, the brain containing 163 smaller magnetite particles presents a total deposited power even smaller than the dielectric, while the brain containing one single larger magnetite particle may have a larger total deposited power. Practically, among all cases, the larger total power deposition in the magnetic brain happened in Gustav's head. In this case, $0.21 \%$ more total power was dissipated in the magnetic brain versus the non-magnetic one. However, the magnetic brain containing 129 spheres of magnetite showed a $5.7 \%$ lower total power loss than the similar pure dielectric brain model.

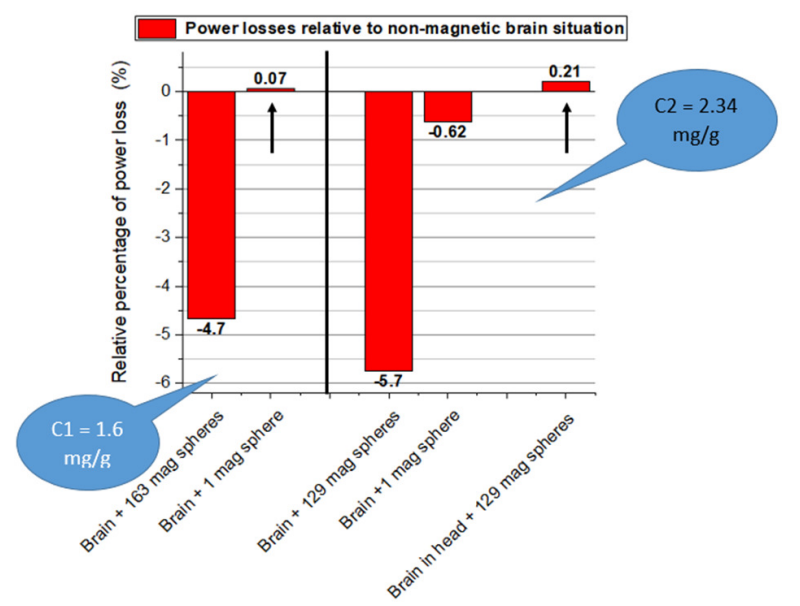

Fig. 12. Relative percentage of total loss deposition in each model: magnetic relative to non-magnetic.

Figure 13 shows comparatively that in most cases the mean power loss density was a little bit higher in the magnetic brain than in the pure dielectric. However, the existence of an exception indicates again the limitations of CST in computing realistically the magnetic losses contribution. Moreover, the larger peak values of power loss density are even more intriguing, as they seem to be present rather in the pure dielectric than in the magnetic brain model.

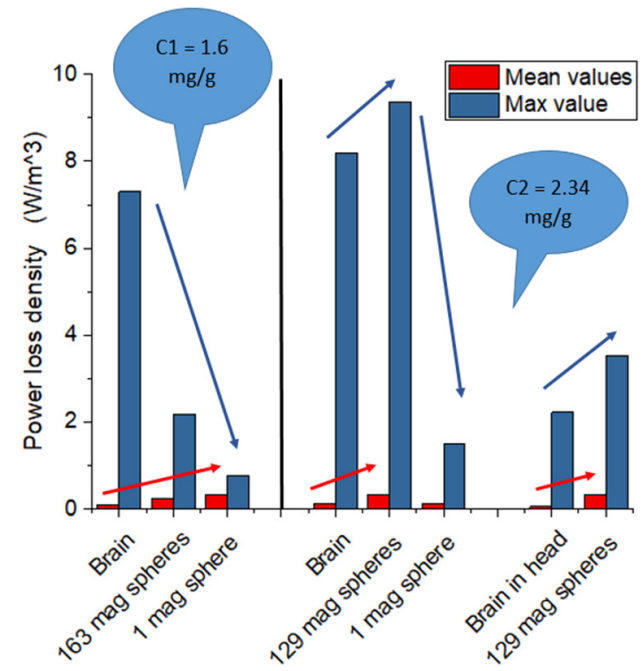

Fig. 13. Average and maximum values of each model's power loss density. 


\section{CONCLUSION}

This paper analyzed a set of simple or respectively anthropomorphic brain models having magnetite content to emphasize the differences in the total power deposited in the tissue when it is considered purely dielectric versus when it is a magnetic-dielectric, with the limitations and the gaps of the dosimetric codes. A patch antenna emitting at $3.5 \mathrm{GHz}$ was modeled and positioned $45 \mathrm{~cm}$ in front of the brain model. Two concentration amounts of magnetite were examined, while its distribution in the brain tissue was modeled either as a unique sphere or a spread of $1 \mathrm{~mm}$ radius particles having the same total volume. Six dosimetric situations were analyzed comparatively, whereas two of them consisted in the brain belonging to the Gustav human head voxel model.

The results showed both higher and lower total power losses in the magnetic- versus non-magnetic brain models. The relative percentage of the losses on the pure dielectric brain was in the range from -5.7 to $+0.21 \%$. The larger the magnetite concentration, the larger the relative differences. If power loss density was normalized to the average E-field strength in a coronal plane tangential to the model, in air, it increased in the case of the magnetic brain of the anthropomorphic model versus the pure dielectric one. The average total SAR normalized to the E-field strength in air showed again that the contribution of the magnetic loss is not consistent when the model is changed. Practically, no clear conclusions could be extracted in connection with the magnetic loss contribution to the whole dissipated power. The results of the mean values of total power loss density showed however more reliability, as the stored magnetic energy density was in all cases dominated by the magnetic brains. Overall, the present approach aimed at underlining the difficulty in getting an accurate solution when requesting absorbed doses of electromagnetic energy in tissues that are not pure dielectrics but have magnetic features. The current computational dosimetry is limited and can only cast a shadow of knowledge about these phenomena. Since experimental dosimetry in the human brain is precluded, it would be of great importance to obtain realistic computational information about the degree of significance that magnetic particles have when they are present in dielectrics and their interaction with signals used by various wireless devices.

\section{REFERENCES}

[1] S. Miclaus, M. Racuciu, and and P. Bechet, "H -Field Contribution to the Electromagnetic Energy Deposition in Tissues Similar to the Brain But Containing Ferrimagnetic Particles, During Use of Face-Held Radio Transceivers," Progress In Electromagnetics Research B, vol. 73, pp. 49-60, 2017, https://doi.org/10.2528/PIERB17010101.

[2] S. Miclaus, C. Iftode, and and A. Miclaus, "Would the Human Brain Be Able to Erect Specific Effects Due to the Magnetic Field Component of an UHF Field via Magnetite Nanoparticles?," Progress In Electromagnetics Research M, vol. 69, pp. 23-36, 2018, https://doi.org/ 10.2528/PIERM18030806.

[3] J. L. Kirschvink, A. Kobayashi-Kirschvink, and B. J. Woodford, "Magnetite biomineralization in the human brain.," Proceedings of the National Academy of Sciences, vol. 89, no. 16, pp. 7683-7687, Aug. 1992, https://doi.org/10.1073/pnas.89.16.7683.

[4] B. A. Maher et al., "Magnetite pollution nanoparticles in the human brain," Proceedings of the National Academy of Sciences, vol. 113, no. 39, pp. 10797-10801, Sep. 2016, https://doi.org/10.1073/pnas. 1605941113.
[5] L. Calderón-Garcidueñas et al., "Combustion- and friction-derived magnetic air pollution nanoparticles in human hearts," Environmental Research, vol. 176, Sep. 2019, Art. no. 108567, https://doi.org/10.1016/ j.envres.2019.108567.

[6] D. Lu et al., "Chemical multi-fingerprinting of exogenous ultrafine particles in human serum and pleural effusion," Nature Communications, vol. 11 , no. 1 , May 2020 , Art. no. 2567 , https://doi.org/10.1038/s41467020-16427-x.

[7] R. Gieré, "Magnetite in the human body: Biogenic vs. anthropogenic," Proceedings of the National Academy of Sciences, vol. 113, no. 43, pp. 11986-11987, Oct. 2016, https://doi.org/10.1073/pnas.1613349113.

[8] Q. Zhang et al., "Separation and Tracing of Anthropogenic Magnetite Nanoparticles in the Urban Atmosphere," Environmental Science \& Technology, vol. 54, no. 15, pp. 9274-9284, Aug. 2020, https://doi.org/ 10.1021/acs.est.0c01841.

[9] J. L. Kirschvink, "Microwave absorption by magnetite: A possible mechanism for coupling nonthermal levels of radiation to biological systems," Bioelectromagnetics, vol. 17, no. 3, pp. 187-194, 1996, https://doi.org/10.1002/(SICI)1521-186X(1996)17:3<187::AID-BEM4> 3.0.CO;2-\#.

[10] F. C. Størmer, "Magnetite in dura and pia mater in human brain. A shield against electromagnetic radiation?," Medical Hypotheses, vol. 82, no. 1, Jan. 2014, Art. no. 123, https://doi.org/10.1016/j.mehy.2013. 11.010.

[11] P. C. Fannin, I. Malaescu, C. N. Marin, and N. Stefu, "Microwave propagation parameters in magnetic fluids," The European Physical Journal E, vol. 29, no. 3, pp. 299-303, Jul. 2009, https://doi.org/ 10.1140/epje/i2009-10477-7.

[12] P. C. Fannin, C. N. Marin, I. Malaescu, and N. Stefu, "Microwave dielectric properties of magnetite colloidal particles in magnetic fluids," Journal of Physics: Condensed Matter, vol. 19, no. 3, Jan. 2007, Art. no. 036104, https://doi.org/10.1088/0953-8984/19/3/036104.

[13] E. M. Alfsen, F. C. Størmer, A. Njå, and L. Walløe, "A proposed tandem mechanism for memory storage in neurons involving magnetite and prions," Medical Hypotheses, vol. 119, pp. 98-101, Oct. 2018, https://doi.org/10.1016/j.mehy.2018.07.003.

[14] M. A. Busquets, A. Espargaró, R. Sabaté, and J. Estelrich, "Magnetic Nanoparticles Cross the Blood-Brain Barrier: When Physics Rises to a Challenge," Nanomaterials, vol. 5, no. 4, pp. 2231-2248, Dec. 2015, https://doi.org/10.3390/nano5042231.

[15] H. Nittby et al., "Nonthermal GSM RF and ELF EMF effects upon rat BBB permeability," The Environmentalist, vol. 31, no. 2, pp. 140-148, Jun. 2011, https://doi.org/10.1007/s10669-011-9307-z.

[16] S. Khan and D. Cohen, "Using the magnetoencephalogram to noninvasively measure magnetite in the living human brain," Human Brain Mapping, vol. 40, no. 5, pp. 1654-1665, 2019, https://doi.org/ 10.1002/hbm. 24477 .

[17] J. Hammond, B. A. Maher, I. A. M. Ahmed, and D. Allsop, "Variation in the concentration and regional distribution of magnetic nanoparticles in human brains, with and without Alzheimer's disease, from the UK," Scientific Reports, vol. 11, no. 1, Apr. 2021, Art. no. 9363, https://doi.org/10.1038/s41598-021-88725-3.

[18] International Commission on Non-Ionizing Radio Protection, "Guidelines for Limiting Exposure to Time-varying Electric, Magnetic, and Electromagnetic Fields (up to 300GHz)," Health Physics, vol. 74, no. 4, pp. 494-522, Apr. 1998.

[19] "IEEE C95.1-2019 - IEEE Standard for Safety Levels with Respect to Human Exposure to Electric, Magnetic, and Electromagnetic Fields, 0 $\mathrm{Hz}$ to $300 \mathrm{GHz}$ " [Online]. Available: https://standards.ieee.org/ standard/C95_1-2019.html.

[20] I. Belyaev, "Duration of Exposure and Dose in Assessing Nonthermal Biological Effects of Microwaves," in Dosimetry in Bioelectromagnetics, Boca Raton, FL, USA: CRC Press, 2017.

[21] A. A. Pilla, "Nonthermal electromagnetic fields: From first messenger to therapeutic applications," Electromagnetic Biology and Medicine, vol. 32, no. 2, pp. 123-136, Jun. 2013, https://doi.org/10.3109/15368378. 2013.776335 . 
[22] L. B. Salford, H. Nittby, and B. R. R. Persson, "Effects of Electromagnetic Fields From Wireless Communication upon the BloodBrain Barrier," in A Rationale for Biologically-Based Exposure Standards for Low-Intensity Electromagnetic Radiation (section 10), BioInitiative Working Group, 2012.

[23] P. Shrivastava and T. R. Rao, "Investigations of SAR Distributions and Temperature Elevation on Human Body at $60 \mathrm{GHz}$ with Corrugated Antipodal Linear Tapered Slot Antenna," Progress In Electromagnetics Research $M$, vol. 59, pp. 111-121, 2017, https://doi.org/10.2528/ PIERM17041707.

[24] T. Hamed and M. Maqsood, "SAR Calculation \& Temperature Response of Human Body Exposure to Electromagnetic Radiations at 28, 40 and $60 \mathrm{GHz}$ mmWave Frequencies," Progress In Electromagnetics Research $M$, vol. 73, pp. 47-59, 2018, https://doi.org/10.2528/PIERM18061102.

[25] J. Lan, T. Hong, X. Liang, and and G. Du, "Evaluation of Microwave Microdosimetry for Human Eyes with Glasses Exposed to Wireless Eyewear Devices at Phone Call State," Progress In Electromagnetics Research M, vol. 63, pp. 71-81, 2018, https://doi.org/10.2528/ PIERM17080802.

[26] K. Y. Yazdandoost and I. Laakso, "Numerical Modeling of Electromagnetic Field Exposure from 5G Mobile Communications at 10 GHz," Progress In Electromagnetics Research M, vol. 72, pp. 61-67, 2018, https://doi.org/10.2528/PIERM18070503.

[27] M. Munde, A. Nandgaonkar, and and S. Deosarkar, "Low Specific Absorption Rate Antenna Using Electromagnetic Band Gap Structure for Long Term Evolution Band 3 Application," Progress In Electromagnetics Research $M$, vol. 80, pp. 23-34, 2019, https://doi.org/ 10.2528/PIERM18102103.

[28] M. Munde, A. Nandgaonkar, and and S. Deosarkar, "Dual Feed Wideband Annular Ring Microstrip Antenna with Circular DGS for Reduced SAR," Progress In Electromagnetics Research B, vol. 88, pp. 175-195, 2020, https://doi.org/10.2528/PIERB20071804.

[29] W. E. May, I. Sfar, J. M. Ribero, and and L. Osman, "Design of LowProfile and Safe Low SAR Tri-Band Textile EBG-Based Antenna for IoT Applications," Progress In Electromagnetics Research Letters, vol. 98, pp. 85-94, 2021, https://doi.org/10.2528/PIERL21051107.

[30] K. Mahmoud, A. Baz, W. Alhakami, H. Alhakami, and and A. M. Montaser, "The Performance of Circularly Polarized Phased Sub-Array Antennas for 5G Laptop Devices Investigating the Radiation Effects," Progress In Electromagnetics Research C, vol. 110, pp. 267-283, 2021, https://doi.org/10.2528/PIERC21012005.

[31] G. M. J. V. Leeuwen, J. J. W. Lagendijk, B. J. A. M. V. Leersum, A. P. M. Zwamborn, S. N. Hornsleth, and A. N. T. J. Kotte, "Calculation of change in brain temperatures due to exposure to a mobile phone," Physics in Medicine and Biology, vol. 44, no. 10, pp. 2367-2379, Aug. 1999, https://doi.org/10.1088/0031-9155/44/10/301.

[32] P. Bernardi, M. Cavagnaro, S. Pisa, and E. Piuzzi, "Specific absorption rate and temperature increases in the head of a cellular-phone user," IEEE Transactions on Microwave Theory and Techniques, vol. 48, no. 7, pp. 1118-1126, Jul. 2000, https://doi.org/10.1109/22.848494.

[33] A. Drossos, V. Santomaa, and N. Kuster, "The dependence of electromagnetic energy absorption upon human head tissue composition in the frequency range of $300-3000 \mathrm{MHz}$, IEEE Transactions on Microwave Theory and Techniques, vol. 48, no. 11, pp. 1988-1995, Nov. 2000, https://doi.org/10.1109/22.884187.

[34] A. Hirata and T. Shiozawa, "Correlation of maximum temperature increase and peak SAR in the human head due to handset antennas," IEEE Transactions on Microwave Theory and Techniques, vol. 51, no. 7, pp. 1834-1841, Jul. 2003, https://doi.org/10.1109/TMTT.2003. 814314.

[35] A. Hirata and T. Shiozawa, "Correlation of maximum temperature increase and peak SAR in the human head due to handset antennas," IEEE Transactions on Microwave Theory and Techniques, vol. 51, no. 7, pp. 1834-1841, Jul. 2003, https://doi.org/10.1109/TMTT.2003. 814314.

[36] A. Hirata, M. Fujimoto, T. Asano, J. Wang, O. Fujiwara, and T. Shiozawa, "Correlation between maximum temperature increase and peak SAR with different average schemes and masses," IEEE
Transactions on Electromagnetic Compatibility, vol. 48, no. 3, pp. 569 578, Aug. 2006, https://doi.org/10.1109/TEMC.2006.877784.

[37] T. Samaras, E. Kalampaliki, and J. N. Sahalos, "Influence of Thermophysiological Parameters on the Calculations of Temperature Rise in the Head of Mobile Phone Users," IEEE Transactions on Electromagnetic Compatibility, vol. 49, no. 4, pp. 936-939, Nov. 2007, https://doi.org/10.1109/TEMC.2007.908257.

[38] A. Hirata and O. Fujiwara, "The correlation between mass-averaged SAR and temperature elevation in the human head model exposed to RF near-fields from 1 to $6 \mathrm{GHz}$," Physics in Medicine and Biology, vol. 54, no. 23, pp. 7227-7238, Nov. 2009, https://doi.org/10.1088/00319155/54/23/013.

[39] I. Laakso, R. Morimoto, A. Hirata, and T. Onishi, "Computational Dosimetry of the Human Head Exposed to Near-Field Microwaves Using Measured Blood Flow," IEEE Transactions on Electromagnetic Compatibility, vol. 59, no. 2, pp. 739-746, Apr. 2017, https://doi.org/ 10.1109/TEMC.2016.2633326.

[40] R. Morimoto, I. Laakso, V. D. Santis, and A. Hirata, "Relationship between peak spatial-averaged specific absorption rate and peak temperature elevation in human head in frequency range of $1-30 \mathrm{GHz}$," Physics in Medicine and Biology, vol. 61, no. 14, pp. 5406-5425, Jul. 2016, https://doi.org/10.1088/0031-9155/61/14/5406.

[41] CST Studio Suite (2019). CST Microwave Studio.

[42] S. Ghnimi, A. Nasri, and A. Gharsallah, "Study of a New Design of the Planar Inverted-F Antenna for Mobile Phone Handset Applications," Engineering, Technology \& Applied Science Research, vol. 10, no. 1, pp. 5270-5275, Feb. 2020, https://doi.org/10.48084/etasr.3287.

[43] S. K. Bitra and S. Miriyala, "An Ultra-Wideband Band Pass Filter using Metal Insulator Metal Waveguide for Nanoscale Applications," Engineering, Technology \& Applied Science Research, vol. 11, no. 3, pp. 7247-7250, Jun. 2021, https://doi.org/10.48084/etasr.4194.

[44] K. Mekki, O. Necibi, C. Boussetta, and A. Gharsallah, "Miniaturization of Circularly Polarized Patch Antenna for RFID Reader Applications," Engineering, Technology \& Applied Science Research, vol. 10, no. 3, pp. 5655-5659, Jun. 2020, https://doi.org/10.48084/etasr.3445.

[45] M. Jazirehpour and S. A. Seyyed Ebrahimi, "Effect of aspect ratio on dielectric, magnetic, percolative and microwave absorption properties of magnetite nanoparticles," Journal of Alloys and Compounds, vol. 638, pp. 188-196, Jul. 2015, https://doi.org/10.1016/j.jallcom.2015.03.021.

[46] M. Jazirehpour and S. A. S. Ebrahimi, "Synthesis of magnetite nanostructures with complex morphologies and effect of these morphologies on magnetic and electromagnetic properties," Ceramics International, vol. 42, no. 15, pp. 16512-16520, Nov. 2016, https://doi.org/10.1016/j.ceramint.2016.07.067.

[47] H. Khurshid et al., "Anisotropy effects in magnetic hyperthermia: A comparison between spherical and cubic exchange-coupled $\mathrm{FeO} / \mathrm{Fe} 3 \mathrm{O} 4$ nanoparticles," Journal of Applied Physics, vol. 117, no. 17, May 2015, Art. no. 17A337, https://doi.org/10.1063/1.4919250.

[48] X. Liu et al., "Shape-dependent magnetic and microwave absorption properties of iron oxide nanocrystals," Materials Chemistry and Physics, vol. 192, pp. 339-348, May 2017, https://doi.org/10.1016/j. matchemphys.2017.02.012.

[49] S. Couture, X. Wang, A. Goncharov, and V. Lomakin, "A coupled micromagnetic-Maxwell equations solver based on the finite element method," Journal of Magnetism and Magnetic Materials, vol. 493, Jan. 2020, Art. no. 165672, https://doi.org/10.1016/j.jmmm.2019.165672.

[50] J. Leliaert and J. Mulkers, "Tomorrow's micromagnetic simulations," Journal of Applied Physics, vol. 125, no. 18, May 2019, Art. no. 180901, https://doi.org/10.1063/1.5093730.

[51] S. Couture and V. Lomakin, "Electromagnetic-micromagnetic simulator for magnetization-eddy current dynamics in magnetic materials and devices," in 2017 IEEE International Symposium on Antennas and Propagation USNC/URSI National Radio Science Meeting, Jul. 2017, pp. 1117-1118, https://doi.org/10.1109/APUSNCURSINRSM.2017. 8072601.

[52] Z. Yao, R. U. Tok, T. Itoh, and Y. E. Wang, "A Multiscale Unconditionally Stable Time-Domain (MUST) Solver Unifying Electrodynamics and Micromagnetics," IEEE Transactions on 
Microwave Theory and Techniques, vol. 66, no. 6, pp. 2683-2696, Jun. 2018, https://doi.org/10.1109/TMTT.2018.2825373.

[53] H. Mosallaei and K. Sarabandi, "Magneto-dielectrics in electromagnetics: concept and applications," IEEE Transactions on Antennas and Propagation, vol. 52, no. 6, pp. 1558-1567, Jun. 2004, https://doi.org/10.1109/TAP.2004.829413.

[54] Q. Nguyen and A. I. Zaghloul, "Susceptibility of Nanoparticles Studied by Landau-Lifshitz-Gilbert and Snoek's Equations," in 2019 IEEE International Symposium on Antennas and Propagation and USNCURSI Radio Science Meeting, Jul. 2019, pp. 1299-1300, https://doi.org/10.1109/APUSNCURSINRSM.2019.8888631.

[55] J. Leliaert, A. Vansteenkiste, A. Coene, L. Dupré, and B. Van Waeyenberge, "Vinamax: a macrospin simulation tool for magnetic nanoparticles," Medical \& Biological Engineering \& Computing, vol. 53, no. 4, pp. 309-317, Apr. 2015, https://doi.org/10.1007/s1 1517-014$1239-6$.

[56] Ansys HFSS - 3D High Frequency Electromagnetic Simulation Software. Ansys.

[57] I. Kong, S. Hj Ahmad, M. Hj Abdullah, D. Hui, A. Nazlim Yusoff, and D. Puryanti, "Magnetic and microwave absorbing properties of magnetite-thermoplastic natural rubber nanocomposites," Journal of Magnetism and Magnetic Materials, vol. 322, no. 21, pp. 3401-3409, Nov. 2010, https://doi.org/10.1016/j.jmmm.2010.06.036.

[58] "Tissue dielectric properties," IT'IS Foundation. https://itis. swiss/virtualpopulation/tissue-properties/database/dielectric-properties (accessed Sep. 28, 2021). 